\title{
A contribution to the pathobiology of Biomphalaria glabrata hemocytes
}

\author{
Claudia Maria da Cunha Borges, Carine Machado Azevedo, Zilton A Andrade ${ }^{+}$
}

Laboratório de Patologia Experimental, Centro de Pesquisas Gonçalo Moniz-Fiocruz, Rua Valdemar Falcão 121, 40295-001

Salvador, BA, Brasil

This study attempts to investigate the relationship between the hemocytes in the two compartments: circulating peripheral lymph and the connective tissues. The hemocytes are compared with the vertebrate macrophages and constitute the principal line of defense against external aggression. The hemocytes were counted in circulating hemolymph and their phagocytic capability was evaluated in Schistosoma mansoni-infected Biomphalaria glabrata and the results were compared with those obtained from normal intact control snails. Although the number of circulating hemocytes revealed a mild increase in snails at the 6th week of infection, the overall findings were similar and pointed out that the cells in the two compartments are not functionally connected. However, the hemocytes found within the connective tissues of infected snails showed definite ultrastructural differences in the number and disposition of cytoplasmic prolongations and organelles in comparison with the hemocytes from non-infected snails. Histochemically, the staining for acid phosphatase activity served as a marker to hemocytes, sometimes being found in extracellular material at the foci of parasite-hemocyte interactions.

Key words: Biomphalaria glabrata - Schistosoma mansoni - hemocytes

During the interactions between the larval forms of the Schistosoma mansoni and its intermediate host Biomphalaria glabrata a pivot role is played by the snail defense cells - the hemocytes. These mobile phagocyte cells have been compared with the vertebrate macrophages, but although the similarities are many, there are also striking differences. It seems that the hemocytes are focally mobilized and act without cooperation from other cells or humoral factors. They are found in the circulating hemolymph and within the connective tissues, but the relationships between these two compartments are poorly understood. Abdul-Salam and Michelson (1980) observed an increase in the number of circulating hemocytes in $B$. glabrata after a 4 and 6-week $S$. mansoni infection. The same was also seen in B. glabrata during infection with Echinostoma liei (Mounkassa \& Jourdane 1990). However, several factors may interfere with these results, such as the age of the snails (Stump \& Gilbertson 1978) and even the temperature of the water were they are maintained (Feng 1965).

On the other hand, hemocytes represent cells with variable morphology and enzymatic contents, with subpopulations exhibiting different abilities for adhesion and phagocytosis (Van der Knaap \& Loker 1990, MatriconGondran \& Letocard 1999).

The present investigation considers the problems above mentioned and look for a correlation between the presence of hemocytes in tissue lesions and in the circulating hemolymph in B. glabrata infected with $S$. mansoni,

\footnotetext{
Financial support: Capes

+Corresponding author: zilton@cpqgm.fiocruz.br

Received 25 May 2006

Accepted 26 June 2006
}

and observes the main histological, histochemical and ultra structural features of the hemocytes within the snail tissues, in an attempt for a better understanding of its patho-biology.

\section{MATERIALS AND METHODS}

Groups and procedures - A stock of snails, originated from the region of Feira de Santana, BA, maintained at the Gonçalo Moniz Research Center for more that 20 years, has been used throughout the experiments. They measured from 8 to $14 \mathrm{~mm}$ in diameter and were maintained under controlled conditions of room temperature $\left(26^{\circ} \mathrm{C}\right)$ with free access to food. They were separated into two groups:

Group 1: normal, intact snails (Controls -8 to $14 \mathrm{~mm}$ )

Group 2: snails submitted to infection with 20 miracidia, which were freshly obtained from the livers of mice infected with the Feira de Santana strain (Andrade \& Sadigursky 1985) of S. mansoni. Thirty to 63 days later the snails were tested for cercarial elimination. The positive ones were transferred to individual recipients and maintained in the dark until the moment for another test or to be killed.

Counting of circulating hemocytes - Snails from both groups were submitted to this procedure. They had their shell perforated with a $26-\mathrm{G} 1 / 2$ insulin needle at the digestive gland level and the emerging hemolymph was aspirated, placed in siliconized tubes (Vacuum II Labnew ${ }^{\circledR}$ ) and maintained at $4^{\circ} \mathrm{C}$. A total of hemolymph $30 \mu 1$ to 100 $\mu 1$ of was collected from each snail. Cell count was performed in a mixture of $10 \mu \mathrm{l}$ hemolymph with a $5 \%$ neutral red solution, and examined in Neubauer chamber. Redstained cells were identified as granulocytes and the nonstained ones as hyalinocytes (Bezerra et al. 1997).

The phagocytosis activity of the circulating hemocytes was also evaluated in these snails. To samples 
of hemolymph containing $10^{5}$ cells and hemolymph soluble factors, it was added $40 \mu \mathrm{l}$ of RPMI-1640 medium, Zimozan A $(0,624 \mathrm{mg} / \mathrm{ml})$ and diluted Indian ink in RPMI $(1: 1000)$. After $3 \mathrm{~h}$ incubation in a water bath at $37^{\circ} \mathrm{C}$, it was added $5 \mathrm{mg} / \mathrm{ml}$ PBS, pH 7.2, of MTT (3-4,5 dimetil tetrazolium bromide, Sigma, US), and the water bath continued for more $2 \mathrm{~h}$, at $37^{\circ} \mathrm{C}$. After that, $1 \mathrm{ml}$ of isopropanol- $\mathrm{HCl} 0,04$ $\mathrm{N}$ was added, homogenized, centrifuged and the supernatant analyzed with a Hitachi U-2000 spectophotomer, under $570 \mathrm{~nm}$ wave-length (Bezerra et al. 1997).

Histopathology - Snails were anesthetized with menthol crystals during $4 \mathrm{~h}$, and the soft parts were removed from the crushed shells and fixed either in Bouin fluid. Five to 10 micrometer thick sections were stained with Hematoxilyn and eosin, PAS-Schiff for sugars, and Siriusred for collagen. Hemocyte reactions were considered as focal (granulomatous) or diffuse, and a semi-quantitative estimation of their grades as mild, moderate and intense was made by two independent observers.

Electron microscopy - Tiny fragments taken from several regions of both infected and non-infected snails, were immediately fixed in $2 \%$ glutaraldehyde in $0.2 \mathrm{M}$ cacodylate buffer, $\mathrm{pH} 7.4$, and postfixed with $1 \%$ osmium tetroxide. Fragments were dehydrated in graded concentrations of acetone and embedded in Poly-bed 812 (Embedding Media Polysciences, IVC). Selected ultrathin sections (50$70 \mathrm{~nm}$ ) were made with a Reichert (Ultratome Supernova Leica) ultramicrotome and mounted on uncoated copper grids, contrasted with uranyl acetate and lead citrate. Specimens were examined in a transmission Zeiss EM-9 electron microscope, which was operated at an acceleration voltage of $50 \mathrm{kV}$.

Histochemistry - The infected snails were killed at 42, 49, and 63 days following miracidial exposition for the histochemical demonstration of two enzymes: one representative of the respiratory chain - dehydrogenase; and a lysossome enzyme - acid phosphatase. After removal of the soft parts, fresh fragments were immediately taken from the regions of digestive glands and ovo-testis and immersed in Tissue-tek (Sakura, Japan) and snap frozen with liquid nitrogen and preserved in air-tight bottles in a freezer at $-70^{\circ} \mathrm{C}$ until the moment the blocks were cut in a Reichert-Jung ( 2.800 Frigocut) cryostat, at $-21^{\circ} \mathrm{C}$.

Acetone-fixed cryostat sections were submitted to a tetrazolium histochemical method for the demonstration of dehydrogenase, using di-sodium succinate as substrate (Pearse 1960).

The presence of acid phosphatase was revealed by using Naphtol AS-BI (BioChemika - 70491, US) as substrate, and pararosaniline as indicator, according to the method described by Barka (1960).

Statistical analysis - A one-way ANOVA test followed by a Neuman-Keuls Multiple Comparison Test was used to compare the data from hemocyte counting and phagocytosis activity analysis.

\section{RESULTS}

The snail infection rate reached $80 \%$, with cercarial elimination varying from 50 to 80 per snail, without much variation with time after exposure to miracidia. The smaller snails (8-11 mm shell diameter) presented slightly, but statistically significant, higher elimination in comparison with larger (12-14 mm) adult snails (Fig. 1A).

The counting of hemocytes in the circulating hemolymph revealed a statistically significant increase in number at the 6th week following miracidial exposure, but from then on the variations observed were not significant, even when the comparison included snails from the 7th-9th week after miracidial exposure, and normal intact controls (Fig. 1C).

The histological sections examined disclosed many parasitic forms with variable degree of tissue reaction. The major concentration of parasites appeared at the digestive gland region and the ovotestis, but they could be found throughout the snail tissues. The host reaction was represented by mild to moderate, usually diffuse, and rarely focal hemocyte infiltration around the abundant multiplying $S$. mansoni forms. Only rarely an encapsulating type of reaction was seen surrounding parasite forms. Sometimes sporocysts and developing cercariae were found without any sign of host reaction (Fig. 2).

The histochemical search for enzymatic activity revealed that dehydrogenase was negative from the hemocytes, but was noted in several other snail structures of the digestive glands and ovotestis, although always presenting a rather faint staining. A more strong reaction was seen in the parasites (Fig. 3A). On the contrary, the mark for acid phosphatase activity appeared well distinct. Besides being present in the hemocyte cytoplasm, acid phosphatase was also revealed in several snail cells in the digestive glands and ovotestis, as well as in the schistosome sporocysts and developing cercariae (Fig. 3B). Also, some hemocytes appeared devoid of acid phosphatase. During accumulations of hemocytes around dead or dying parasites a staining for acid phosphatase was sometimes found extra-cellularly, forming a halo around the disintegrating parasite.

Under the electron microscope the hemocytes were frequently found in the connective tissues of the digestive glands and ovotestis. In infected snails the hemocytes presented denser and larger cytoplasm, with many organelles, and more numerous philopodia, when compared with the hemocytes seen in normal snails. Not only dark bodies (lysossomes) and phagosomes (myelin figures), but also mitochondria and granular endoplasmic reticulum appeared more prominent in infected snails when compared with hemocytes from normal snails (Figs 3C, D). The spaces between the numerous cytoplasmic prolongations appeared filled with slightly dense granules. These prolongations formed a characteristic labyrinthine picture. Glycogens granules were also frequentely concentrated in the cytoplasm of hemocytes from infected snails.

\section{DISCUSSION}

The expectation that changes involving the hemocytes within the B. glabrata tissues would have a repercussion on the peripheral hemolymph did not come true. Although the snails used were very susceptible to $S$. mansoni infection, large areas of hemocyte infiltrations did occur in some specimens, but variations in hemocyte counting and in their signs of increased phagocytic activity were al- 
A
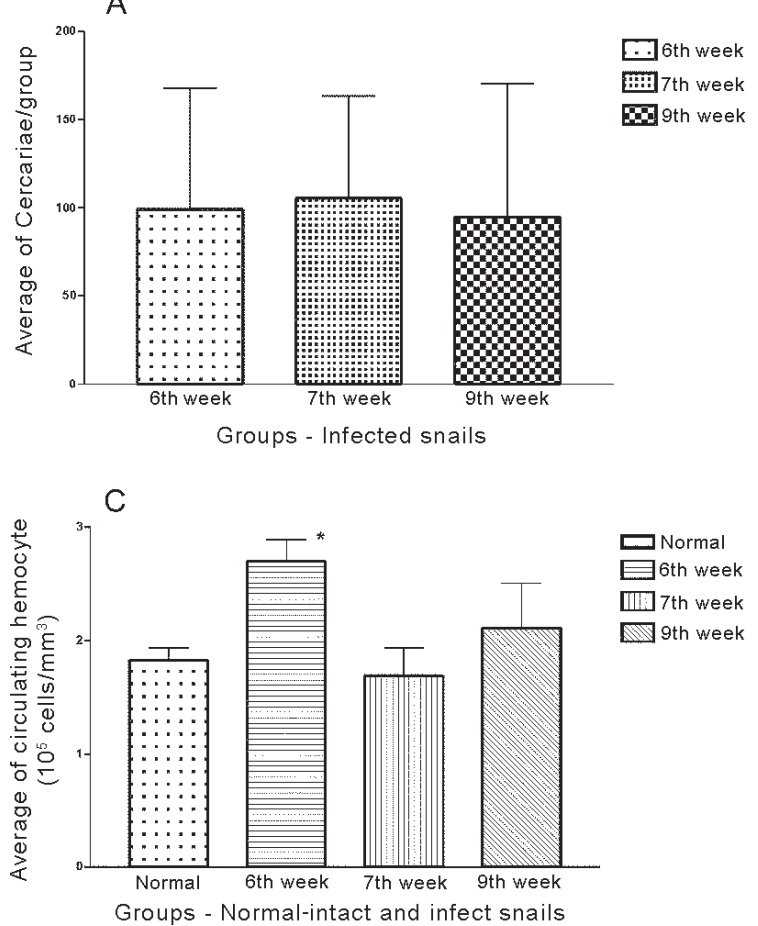

B

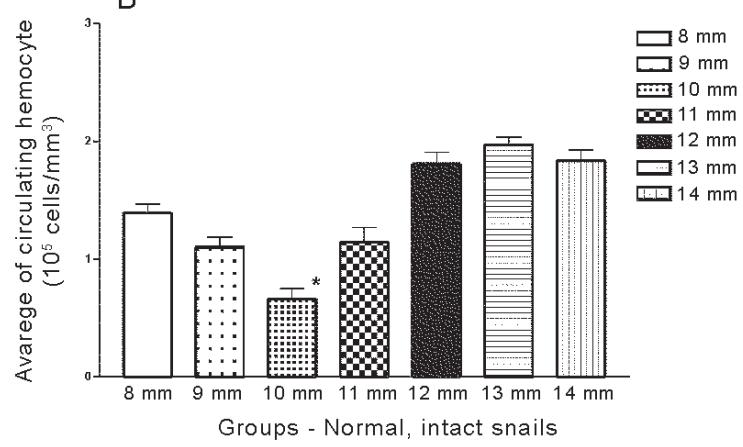

D

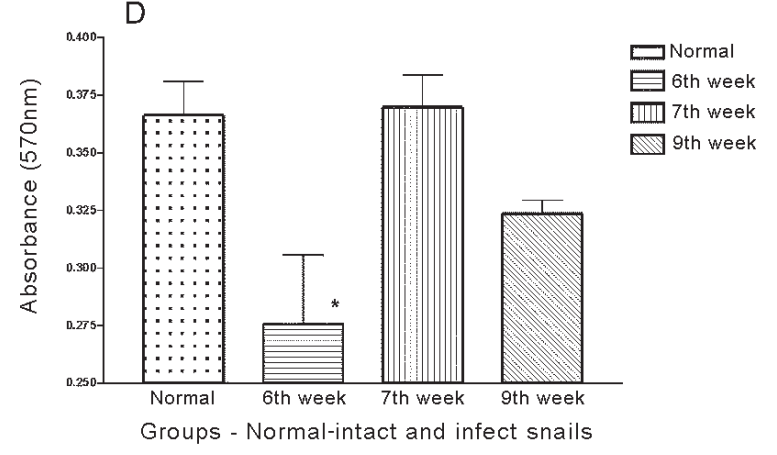

Fig 1A: number of cercariae (average) being eliminated per group of snails at different periods of time after infection with Schistosoma mansoni (6th , 7th and 9th weeks) $(\mathrm{n}=10)$; B: number of circulating hemocytes in hemolymph of normal-intact Biomphalaria glabrata of different sizes; (Sample $=10 \mu 1$ of total hemolymph). Each point represents the average number of cells \pm SD obtained from ten snails per group; asterisk indicates significant differences in hemocytes number as compared with the other groups (one-way ANOVA test and Neuman-Keuls Multiple Comparison Test, $\mathrm{p}<0.001)$; C: number of circulating hemocytes in hemolymph of normal-intact and infected B. glabrata. Infection was made with 20 miracidia of $S$. mansoni (6th, 7 th, and 9 th weeks). Each group with 10 snails, measuring $12-13$ $\mathrm{mm}$ in shell diameter. The points represent the average number of cells $\pm \mathrm{SD}$ expressed as snails per group; asterisk indicates significant differences in hemocyte number as compared with other groups (one-way ANOVA test and Neuman-Keuls Multiple Comparison Test, p < 0.001); D: evaluation of phagocytosis (Zimozan A particles) by circulating hemocytes from normal-intact and infected $B$. glabrata snails; asterisk indicates significant differences in hemocytes number as compared with the other groups (one-way ANOVA test and Neuman-Keuls Multiple Comparison Test, $\mathrm{p}<0.001)$.

ways scarce. Probably the two compartments are independent, and nothing similar to a vertebrate leukocytosis is to be expected during the occurrence of inflammatorylike changes in the snail tissues. Actually, some mild variations did occur in hemocyte counting, but this applied more to the size of the snail (shell diameter), rather than to the presence of schistosome infection. Regarding this latter finding our data only confirm those of Stumpf and Gilbertson (1978), and Dikkeboom et al. (1984). The shell size is related to the age of the snail, and probably the variation in hemocyte concentration in the hemolymph may be under the influence of several factors, among them sexual maturity (Sminia et al. 1974). In the present study, especial attention was paid in order to previously determine the concentration of hemocyte in the hemolymph of normal snails with different ages (shell diameter). It was noted that normal snails measuring $10 \mathrm{~mm}$ of shell diameter presented the lowest hemocyte count, what was probably related to their stage of sexual maturity (Fig. 1B). The stage of the schistosome infection also is important. Abdul-Salam and Michelson (1980) observed significant increase in peripheral hemocyte number in snails with 46-week old infection as compared to normal controls. On the other hand, Granath and Yoshino (1983) found the contrary, a decrease in hemocyte number, that they attributed to a migration of hemolymph cells to the interior of the tissues.

Another tested parameter was phagocytosis activity to zymosan. It did not presented differences when peripheral hemocytes from infected snails were compared to non-infected controls. This has been interpreted as another line of evidences that B. glabrata hemocytes from the lymph and tissues depend on independent and peculiar types of stimulation during $S$. mansoni infection.

B. glabrata hemocytes have frequently been compared with the vertebrate macrophages. The macrophages are cells specially adapted to intracellular digestion. So they frequently present lysossomes ready to pour their digestive enzymes, among them several acid hydrolyses, into phagocytic vacuoles. These aspects have already been studied (Carter \& Bogitsh 1975, Folley \& Cheng 1977, Cheng 1983, Borges \& Andrade 2003). However, two aspects now observed deserve comments. The presence of acid phosphatase activity found free within the tissues, denoting the possibility of this enzyme to act extracellularly, being secreted and eliminated at some reaction zones. 
Another aspect refers to the finding of acid phosphatase positive and negative hemocytes, which suggests cells at different stages of functional activity.

Present ultrastructural findings reveal mild but definite differences between hemocytes present in the tis- sues of infected and non-infected snails. The formal present signs of activation in the number, size and disposition of cytoplasmic prolongations, and in the number and size of organelles. On this later aspect, the tissue hemocytes also differ from their peripheral counterpart.
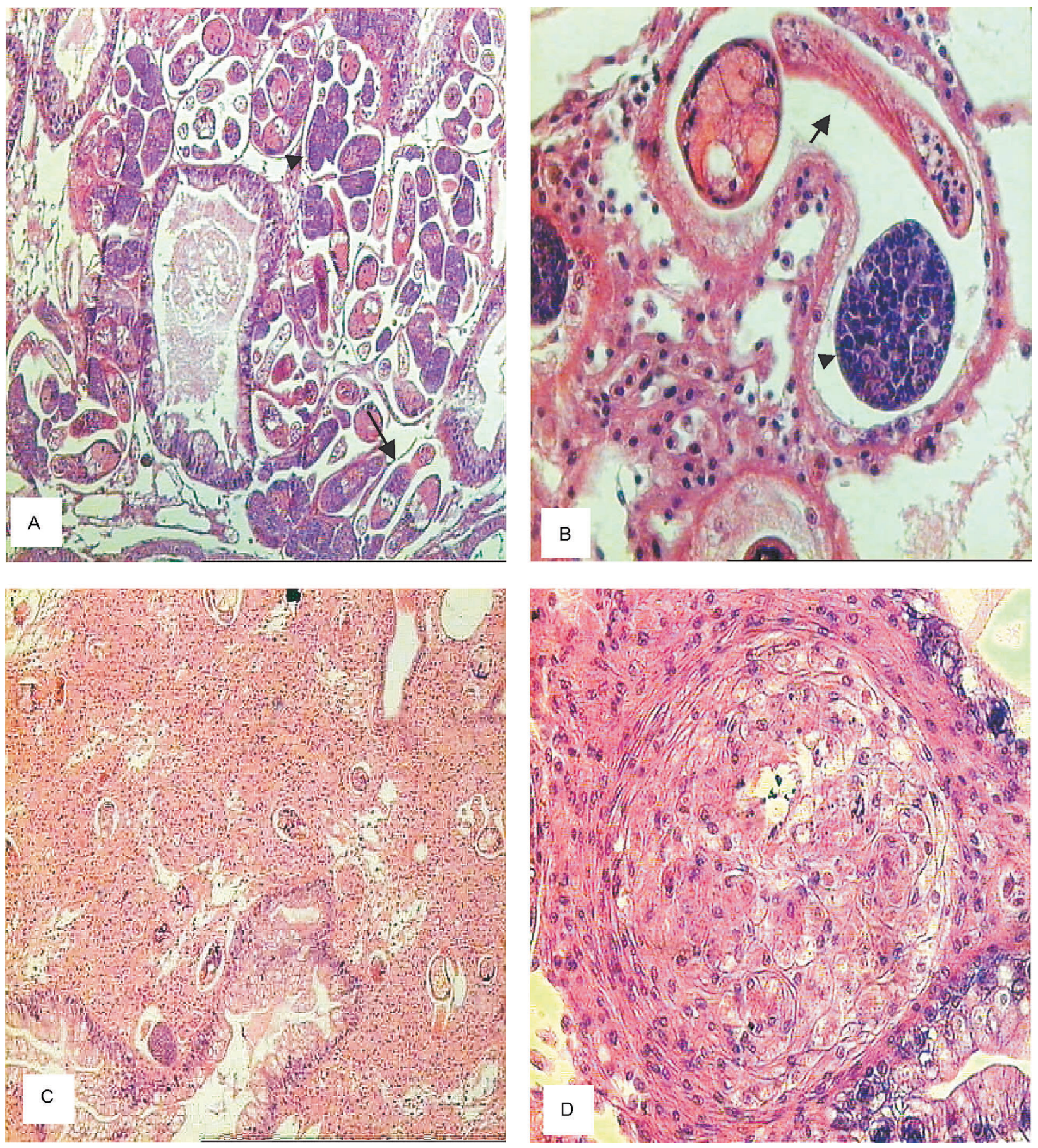

Fig. 2: different aspects of the interactions of hemocytes and Schistosoma mansoni sporocysts and developing cercariae within the tissues of Biomphalaria glabrata. A: numerous sporocysts (arrow-head) and developing cercariae (arrow) with digestive glands in the absence of tissue reaction in a very susceptible snail, 200×; B: detail showing the parasite forms (arrow and arrow-head) in the snail digestive glands, with a mild hemocytic infiltration around, 400x; C: an extensive proliferation of hemocytes dissociating the digestive glands and encircling isolated parasite forms, as seen in a resistant snail, 100x; D: detail of a granuloma-like structure formed by hemocytes of a resistant snail, 400X. All pictures from sections stained with hematoxylin and eosin. 

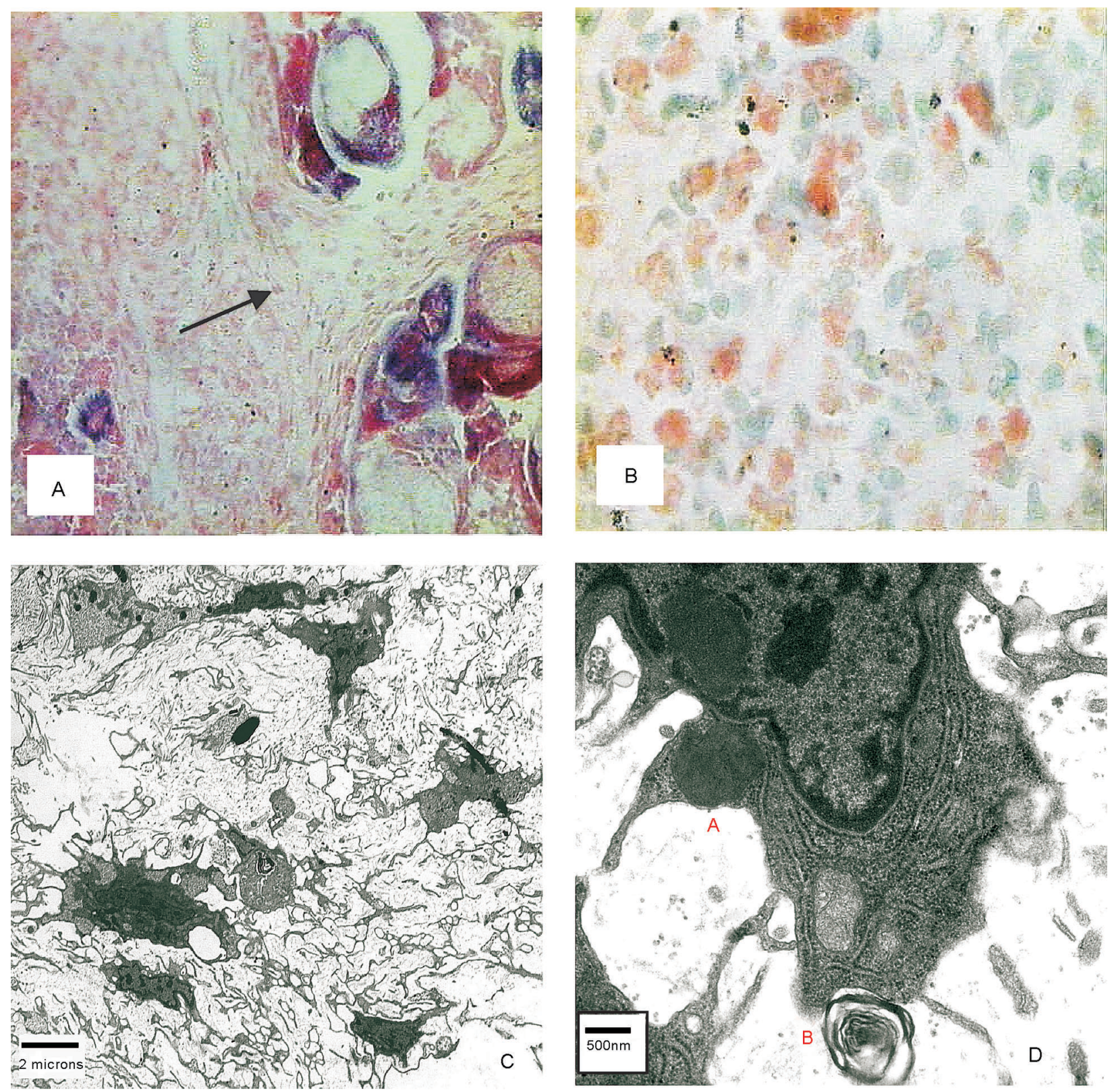

Fig. 3A: histochemical demonstration for succinic dehydrogenase showing activity present in parasite structures, but not in hemocytes. Pearse's method, 200×; B: hemocytes present in the interstitial tissue of the digestive gland some of them showing red-staining acid phosphatase activity. Barka's method, 1000x; C and D: electron micrographs demonstrating hemocytes in infected snails; depicting the abundant cytoplasmic prolongations (C), and details of the rich endoplasmic reticulum, lipid inclusions (A), and an expelled myelin figure (B).

\section{ACKNOWLEDGMENTS}

To Dr Joseph Schrevel chief the Laboratoire de Biologie Parasitaire, Protistologie, Helminthologie (Museum National D\&Histoire Naturelle) for receiving one of us (CMC Borges) in his Laboratory, where the studies with electron microscopy were undertaken.

\section{REFERENCES}

Abdul-Salam JM, Michelson EH 1980. Biomphalaria glabrata amoebocytes: Effect of Schistosoma mansoni infection on in vitro phagocytosis. J Invertebr Pathol 35: 241-248.

Andrade ZA, Sadigursky M 1985. Um estudo comparativo das cepas de Feira de Santana (Bahia) e Porto Rico do Schistosoma mansoni na infecção experimental do camundongo. Mem Inst Oswaldo Cruz 80: 37-40.

Barka T 1960. A simple azo-dye method for histochemical demonstration of acid phosphatase, Nature 187: 248-249.

Bezerra FSM, Nogueira-Machado JA, Chaves MM, Martins RL,Coelho PMZ 1997. Quantification of the population and phagocytary activity of hemocytes of resistant and suceptible of Biomphalaria glabrata and Biomphalaria tenagophila infected with Schistosoma mansoni. Rev Inst Med Trop São Paulo 39: 197-201. 
Borges CMC, Andrade ZA 2003. Extra-cellular Matrix Changes in Schistosoma mansoni-infected Biomphalaria glabrata. Mem Inst Oswaldo Cruz 98: 135-139.

Carter OS, Bogitsh BJ 1975. Histologic and cytochemical observations of the effects of Schistosoma mansoni on Biomphalaria glabrata. Ann NY Acad Sci 266: 380.

Cheng TF 1983. The role of lysosomes in molluscan inflammation. Amer Zool 23: 129-144.

Dikkeboom R, Van Der Knaap WPW, Meuleman E, Sminia T 1984. Differences between blood cells of juvenile and adult specimens of pond snail Lymnaea stagnalis. Cell Tiss Res 238: 43-47.

Feng SY 1965. Heart rate and leucocyte circulation in Cassostrea virginica (Gmelin). Biol Bull 128: 198-210.

Foley DA, Cheng TC 1977. A quantitative study of phagocytosis by hemolymph cells of the pelecypods Crassostrea virginica and Mercenaria mercenaria. J Invertebr Pathol 25: 189-197.

Granath Jr WO, Yoshino TP 1983. Characterization of molluscan phagocyte subpopulations based on lysosomal enzyme markers. J Exper Zool 226: 205-210.

Matricon-Gondran M, Letocart M 1999. Internal defense of the snail Biomphalaria glabrata. II. In vivo responses of hemocytes and fixed phagocytes to injected foreign materials. J Invertebr Pathol 74: 235-247.

Mounkassa JB, Jourdane J 1990. Dynamics of the leukocytic response of Biomphalaria glabrata during the larval development of Schistosoma mansoni and Echinostoma liei. J Invertebr Pathol 55: 306-311.

Pearse AGE 1960. Histochemistry, Theoretical and Applied, 2nd. ed. Little Brown and Co., Boston, p. 451-910.

Sminia T, Borghart R, Van Der Linde AN 1974. Encapsulation of foreign materials experimentally indroduced into the freshwater snail Lymnaea stagnalis. Cell Tiss Res 153: 307-326.

Stumpf JL, Gilbertson DE 1978. Hemocytes of Biomphalaria glabrata: Factors affecting variability. J Invertebr Pathol 32: 177-181.

Van der Knaap WPW, Loker ES 1990. Immune Mechanisms in Trematode - Snail Interactions. Parasitol Today 6: 175182. 